\title{
Role of Spatial Averaging in the Precision of Gene Expression Patterns
}

\author{
Thorsten Erdmann, ${ }^{1}$ Martin Howard, ${ }^{2}$ and Pieter Rein ten Wolde ${ }^{1}$ \\ ${ }^{1}$ FOM Institute for Atomic and Molecular Physics, Science Park 104, 1098 XG Amsterdam, The Netherlands \\ ${ }^{2}$ Department of Computational and Systems Biology, John Innes Centre, Norwich NR4 7UH, United Kingdom
}

(Received 22 July 2009; published 17 December 2009)

\begin{abstract}
During embryonic development, differentiating cells respond via gene expression to positional cues from morphogen gradients. While gene expression is often highly erratic, embryonic development is precise. We show by theory and simulations that diffusion of the expressed protein can enhance the precision of its expression domain. While diffusion lessens the sharpness of the expression boundary, it also reduces super-Poissonian noise by washing out bursts of gene expression. Balancing these effects yields an optimal diffusion constant maximizing the precision of the expression domain.
\end{abstract}

DOI: 10.1103/PhysRevLett.103.258101

PACS numbers: 87.17.Pq, 87.17.Aa, 87.18.Tt

Embryonic development is driven by orderly, spatial patterns of gene expression that assign each cell in the embryo its particular fate. While gene expression is often highly stochastic, embryonic development is exceedingly precise. A vivid example is the Bicoid-Hunchback system in the early Drosophila embryo. Shortly after fertilization, the morphogen protein Bicoid (Bcd) forms an exponential concentration gradient along the anterior-posterior axis of the embryo, which provides positional information to the differentiating nuclei. One of the target genes of Bcd is hunchback $(h b)$, which is expressed in the anterior half of the embryo. The posterior boundary of the $h b$ expression domain is very sharp: by cell cycle 13 , the position of the boundary varies only by about one nuclear spacing [1-3]. This precision is higher than the best achievable precision for a time-averaging based readout mechanism of the Bcd gradient [2]. Intriguingly, the study of Gregor et al. revealed that the $\mathrm{Hb}$ concentrations in neighboring nuclei exhibit spatial correlations and the authors suggest that this implies a form of spatial averaging enhancing the precision of the posterior $\mathrm{Hb}$ boundary [2]. However, the mechanism for spatial averaging remained unclear.

In this manuscript, we analytically and numerically study the Bcd-Hb system. Our analysis reveals a simple, yet powerful mechanism for spatial averaging, which is based on the diffusion of $\mathrm{Hb}$ itself $[2,4]$. We show analytically that $\mathrm{Hb}$ diffusion between neighboring nuclei reduces the super-Poissonian part of the noise in its concentration, with a factor that depends on the diffusion length of $\mathrm{Hb}$ and the dimensionality of the system. In essence, diffusion reduces the noise by washing out bursts in gene expression. This mechanism is generic, and applies not only to any developmental system, but also to any biochemical network in general. For example, if a signaling protein is activated at one end of the cell and then has to diffuse to another place to activate another system, e.g., the messenger CheY in bacterial chemotaxis, then our results show that the non-Poissonian noise in the activation of the signaling protein is washed out by diffusion; for this reason it may be beneficial to spatially separate the in- and output of a signaling pathway. Our analysis also reveals that, while $\mathrm{Hb}$ diffusion reduces the noise, it also lessens the steepness of its expression boundary [2]. The interplay between these two antagonistic effects leads to an optimal diffusion constant of $D \simeq 0.1 \mu \mathrm{m}^{2} / \mathrm{s}$ that maximizes the precision of the $h b$ expression domain.

Our model of the Bcd-Hb system includes the stochastic and cooperative activation of $\mathrm{Hb}$ by $\mathrm{Bcd}$ in each nucleus, and the diffusion of $\mathrm{Hb}$ between neighboring nuclei. To model the cooperative binding of Bcd to the $h b$ promoter, we assume that Bcd proteins can bind sequentially to five binding sites on the $h b$ promoter. When all five sites are occupied, the promoter is active (activity $n=1$ ) and $\mathrm{Hb}$ proteins are produced stochastically with rate $\beta$; otherwise the promoter is inactive $(n=0)$. Hb proteins are degraded stochastically with rate $\mu$. To obtain a lower bound on the precision of the $h b$ expression domain, we assume that Bcd binds to the promoter with a diffusion-limited on-rate $k_{\mathrm{on}}=4 \pi \alpha D_{\mathrm{B}} / V=24 \alpha D_{\mathrm{B}} / d^{3}=8.4 \times 10^{-5} \mathrm{~s}^{-1}$ where $\alpha \simeq 3 \mathrm{~nm}$ is the dimension of a binding site, $d \simeq 6.5 \mu \mathrm{m}$ the diameter of a nucleus and $D_{\mathrm{B}} \simeq 0.32 \mu \mathrm{m}^{2} / \mathrm{s}$ the Bcd diffusion constant [5]. Since the on rate is assumed to be diffusion limited, cooperativity of $\mathrm{Hb}$ activation is tuned via the Bcd off-rate $k_{\text {off }}(j)=a / b^{j}$ which decreases with increasing number $j$ of promoter-bound Bcd. Using $a=$ $12.3 \mathrm{~s}^{-1}$ and $b=6$ the average promoter activity as a function of the number of Bcd molecules $B$ in the nucleus approximately follows the Hill function

$$
\langle n(B)\rangle \simeq B^{h} /\left(B^{h}+K_{\mathrm{eq}}^{h}\right),
$$

with $h=5$ and $K_{\text {eq }}=690$. The Hill-coefficient $h$ was inferred from the relation between $\mathrm{Hb}$ and Bcd concentration, and the activation threshold $K_{\text {eq }}$, where $\left\langle n\left(K_{\text {eq }}\right)\right\rangle=$ 0.5 , is the average number of Bcd molecules $\langle B\rangle$ in a nucleus at the $\mathrm{Hb}$ domain boundary [2].

To describe the formation of the $\mathrm{Hb}$ pattern, we place $N=64 \times 64=4096$ nuclei on a square lattice with spacing $\ell=8.5 \mu \mathrm{m}$ [2]. Hb diffuses over the lattice with 
diffusion constant $D$. With reflecting boundary conditions in one and periodic in the other direction, our model is a cylinder which is symmetric around the anterior-posterior $x$ axis. Activation of $\mathrm{Hb}$ is induced by the Bcd profile

$$
B(x)=K_{\mathrm{eq}} e^{-\left(x-x_{t}\right) / \lambda_{\mathrm{B}}},
$$

which decreases exponentially with the distance $x$ from the anterior pole at $x=0$. The decay length is $\lambda_{\mathrm{B}}=$ $119.5 \mu \mathrm{m}$ [2] and the threshold position for $\mathrm{Hb}$ activation is set to $x_{t}=0.5 \mathrm{~L} ; L=64 \ell=544 \mu \mathrm{m}$ is the length of the embryo. Diffusion of Bcd between nuclei induces fluctuations in the Bcd copy number on the time scale $\tau_{d}=$ $\ell^{2} /\left(4 D_{\mathrm{B}}\right) \simeq 10^{2} \mathrm{~s}$. Because $\tau_{d}$ is much smaller than the time scale for promoter binding, $k_{\text {on }}^{-1} \simeq 10^{4} \mathrm{~s}$, Bcd copy number fluctuations are efficiently averaged out by slow binding of Bcd to the promoter. We therefore assume that the total number of Bcd in a nucleus is constant and given by Eq. (2). The state of the system is described by the number of promoter-bound $\mathrm{Bcd}$ and the number of $\mathrm{Hb}$ in every nucleus. The reaction-diffusion dynamics is simulated using the next-subvolume algorithm described in [6].

Figure 1(a) shows simulation results for the average number of free Bcd molecules $\langle B\rangle$, the average promoter activity $\langle n\rangle$, and the average number of $\mathrm{Hb}$ molecules $\langle H\rangle$ as function of the position $x / L$. Without $\mathrm{Hb}$ diffusion $\langle H(x)\rangle$ is proportional to $\langle n(x)\rangle$. For a finite $\mathrm{Hb}$ diffusion constant, however, the shape of the Hb profile is determined not only by $\langle n(x)\rangle$, but also by the diffusion length of $\mathrm{Hb}, \lambda=\sqrt{D / \mu}$ - with increasing $\lambda$ the profile becomes less steep. Figure 1(b) shows instantaneous $\mathrm{Hb}$ profiles at different times. Fluctuations of promoter activity induce large fluctuations of $H$, which lead to an uncertainty $\Delta x$ in the position at which $H$ crosses the threshold $\left\langle H\left(x_{t}\right)\right\rangle=$ $0.5 \beta / \mu$.

We now consider the $\mathrm{Hb}$ boundary width $\Delta x$ when the $\mathrm{Hb}$ diffusion constant $D$ is zero. Figure 2(a) shows that this decreases with increasing lifetime of $\mathrm{Hb}$. To understand this, we note that, to a good approximation,

$$
\Delta x=\frac{\sigma\left(x_{t}\right)}{\left|\left\langle H\left(x_{t}\right)\right\rangle^{\prime}\right|},
$$
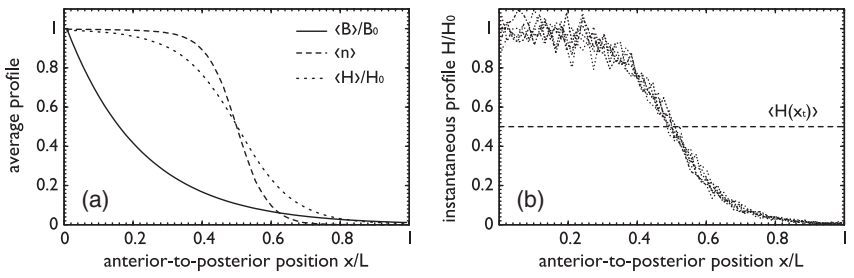

FIG. 1. (a) Normalized average number of free $\mathrm{Bcd},\langle B\rangle / B_{0}$, of $\mathrm{Hb},\langle H\rangle / H_{0}$, and average promoter activity $\langle n\rangle$ in a nucleus as function of the position $x / L ; B_{0}=6480$ and $H_{0}=500 \mathrm{Hb}$ decay rate is $\mu=1.2 \times 10^{-4} \mathrm{~s}^{-1}$, production rate $\beta=500 \mu=$ $0.06 \mathrm{~s}^{-1}$, and $\mathrm{Hb}$ diffusion constant $D=0.32 \mu \mathrm{m}^{2} / \mathrm{s}$. The offrate of the active promoter state is $k_{\text {off }}(5) \simeq 1.6 \times 10^{-3} \mathrm{~s}^{-1}$. (b) Instantaneous $\mathrm{Hb}$ profiles at different times. All simulations are for the full, two-dimensional system. where $\sigma\left(x_{t}\right)$ is the standard deviation of the $\mathrm{Hb}$ copy number and $\left|\left\langle H\left(x_{t}\right)\right\rangle^{\prime}\right|$ is the magnitude of the $\mathrm{Hb}$ gradient at the boundary position $x_{t}[2,7]$. When the Hb lifetime $\tau$ is much longer than the time scale $k_{\text {off }}(5)^{-1}$ of the promoter state fluctuations, then the noise in Hb copy number becomes Poissonian and the variance is given by the mean: $\sigma^{2}\left(x_{t}\right)=\left\langle H\left(x_{t}\right)\right\rangle=\left\langle n\left(x_{t}\right)\right\rangle \beta / \mu=0.5 \beta \tau$. On the other hand, the steepness of the boundary, $\left|\left\langle H\left(x_{t}\right)\right\rangle^{\prime}\right|$, increases linearly with $\tau$ when $D=0$ and the synthesis rate $\beta$ is kept constant. Equation (3) thus predicts that $\Delta x$ decreases as $\tau^{-1 / 2}$ for large $\tau \gg k_{\text {off }}(5)^{-1}$, which is indeed observed in the simulations [see Fig. 2(a)]. Hence, the $h b$ expression boundary could be made arbitrarily precise if the lifetime of $\mathrm{Hb}$ could be increased indefinitely.

In practice the averaging time cannot be made arbitrarily long [2]. Ultimately, it is limited by the nuclear division time $\tau_{\text {nd }}$, which at cell cycle 13 is roughly $30 \mathrm{~min}$. Figure 2(a) shows that $\Delta x \simeq 4$ nuclei for $\tau \simeq \tau_{\text {nd }}$ and $D=$ 0 , which is larger than the precision measured experimentally [2]. The limited averaging time thus puts strong constraints on the precision that can be achieved via the mechanism of time-averaging alone.

Figure 2(a) reveals, however, that the precision of the $h b$ expression domain can be enhanced significantly by increasing the $\mathrm{Hb}$ diffusion constant $D$ to a finite value. This may seem surprising, since the steepness of the boundary, $\left|\left\langle H\left(x_{t}\right)\right\rangle\right|$, decreases with increasing $D$ (Fig. 1) and thisas Eq. (3) shows - tends to increase the boundary width. However, increasing $D$ also reduces the noise in the $\mathrm{Hb}$ copy number. This is the mechanism of spatial averaging, which we now study analytically. Increasing precision by $\mathrm{Hb}$ diffusion was proposed by [2] and demonstrated recently in a large-scale numerical study [4].

To elucidate the mechanism of spatial averaging, we first analyze how the steepness of the $\mathrm{Hb}$ boundary depends on the $\mathrm{Hb}$ diffusion length $\lambda$, and then how the noise in the $\mathrm{Hb}$ copy number at the boundary, $\sigma\left(x_{t}\right)$, depends on $\lambda$ [see Eq. (3)]. To calculate the $\mathrm{Hb}$ profile, we note that a nucleus at a position $x_{i}$ produces $\mathrm{Hb}$ with an average rate $\beta\left\langle n\left(x_{i}\right)\right\rangle$ [Fig. 3(a)]. Analyzing the discrete biological system using a continuum approximation, in one dimension the steady-
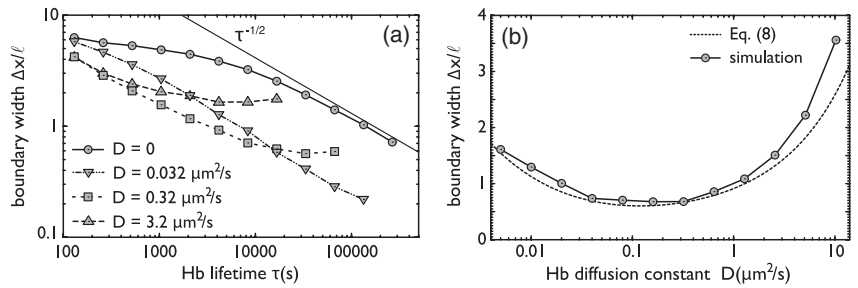

FIG. 2. (a) Simulation results for the Hb boundary width $\Delta x$ as a function of the Hb lifetime $\tau=\mu^{-1}$ for a constant production rate $\beta=0.06 \mathrm{~s}^{-1}$ and different diffusion constants $D$. The solid line scales as $\tau^{-1 / 2}$. (b) Simulation results for $\Delta x$ as a function of $D$ and for $\beta$ and $\mu$ as in Fig. 1. The simulation results are compared to Eq. (3) using Eqs. (5) and (8). All simulations are for the full, two-dimensional system. 
state number of $\mathrm{Hb}$ molecules in a nucleus of size $\ell$ at $x$ due to a point source at $x_{i}$ is

$$
\left\langle H\left(x ; x_{i}\right)\right\rangle_{\mathrm{ps}} \approx \frac{\beta\left\langle n\left(x_{i}\right)\right\rangle / \mu}{2(\lambda / \ell)} e^{-\left|x-x_{i}\right| / \lambda} .
$$

The total $\mathrm{Hb}$ profile is the sum $\langle H(x)\rangle=\sum_{i}\left\langle H\left(x ; x_{i}\right)\right\rangle_{\mathrm{ps}}$ over all point sources $x_{i}=0, \ell, 2 \ell, \ldots$, which is approximated as $\langle H(x)\rangle \approx \int d x^{\prime}\left\langle H\left(x ; x^{\prime}\right)\right\rangle_{\mathrm{ps}} / \ell$. To perform the integration, we approximate the activity $\left\langle n\left(x_{i}\right)\right\rangle$ from Eqs. (1) and (2) by a piecewise linear function which behaves as $\left\langle n\left(x_{i}\right)\right\rangle=0.5+m\left(x_{t}-x_{i}\right)$ around the boundary for $x_{t}-$ $1 / 2 m<x_{i}<x_{t}+1 / 2 m$ and which is unity below and zero above the boundary. The slope $-m=-h / 4 \lambda_{\mathrm{B}}$ is the derivative of $\left\langle n\left(x_{i}\right)\right\rangle$ at $x_{i}=x_{t}$. At the boundary $x_{t}$ the slope of $\langle H(x)\rangle$ is

$$
\left\langle H\left(x_{t}\right)\right\rangle^{\prime}=m \frac{\beta}{\mu}\left\{1-e^{-(1 / 2 m \lambda)}-\sinh \left(\frac{1}{2 m \lambda}\right) e^{-\left(2 x_{t} / \lambda\right)}\right\} .
$$

It increases linearly with the number of $\mathrm{Hb}$ molecules $\beta / \mu$ and decreases as $\lambda$ increases. Because of the rotational symmetry, the slope in the full, two-dimensional system is also given by Eq. (5). Figure 3(b) plots Eq. (5) as function of $\lambda$ and shows that the prediction agrees very well with the two-dimensional simulation results.

Next, we study the effect of $\mathrm{Hb}$ diffusion on the variance in the $\mathrm{Hb}$ copy number $\sigma^{2}$ [see Eq. (3)]. For clarity, we first do the calculations for one dimension and extend our approximation to general dimensions afterwards. To compute $\sigma^{2}$, we exploit the observation made above that each nucleus acts as a point source of $\mathrm{Hb}$ [Fig. 3(a)], and that the expression of $\mathrm{Hb}$ in each nucleus is an independent stochastic process. We denote the variance of the $\mathrm{Hb}$ copy number in a nucleus at a distance $x$ from the point source as $\sigma_{\mathrm{ps}}^{2}(x)$. In a uniform space, the total $\mathrm{Hb}$ variance in a nucleus is the sum $\sigma^{2}=\sum_{i} \sigma_{\mathrm{ps}}^{2}\left(x_{i}\right)$ over all point sources, which is approximated as $\sigma^{2} \approx \int d x \sigma_{\mathrm{ps}}^{2}(x) / \ell$. To calculate $\sigma_{\mathrm{ps}}^{2}(x)$, we assume that the $\mathrm{Hb}$ dynamics is fast on the time scale of promoter switching, such that the $\mathrm{Hb}$ concentra-
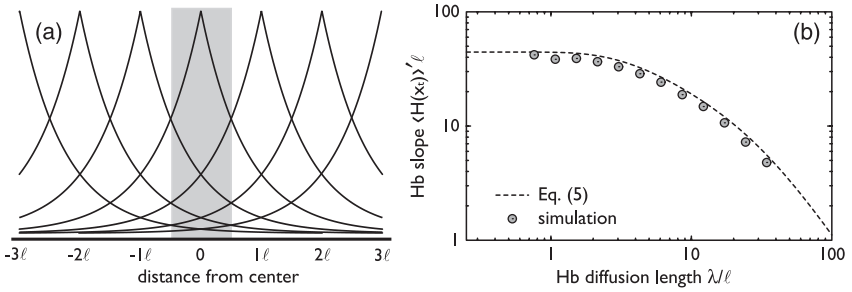

FIG. 3. (a) Hb is produced in nuclei and diffuses away from these point sources. The stationary number of $\mathrm{Hb}$ in a given nucleus (shaded area) is the sum over contributions from all nuclei. (b) Slope $\left\langle H\left(x_{t}\right)\right\rangle^{\prime}$ of the Hb profile at the boundary $x_{t}$ as function of the $\mathrm{Hb}$ diffusion length $\lambda=\sqrt{D / \mu}$. All lengths are in units of the lattice constant $\ell=8.5 \mu \mathrm{m}$. Analytical results from Eq. (5) (dashed line) are compared to simulation results (symbols) for the full, two-dimensional system. tion switches between zero when the promoter is off, and $\langle H(x ; 0)\rangle_{\text {ps }} /\langle n\rangle$, with $\langle H(x ; 0)\rangle_{\text {ps }}$ given by Eq. (4), when the promoter is on. In this limit,

$$
\sigma_{\mathrm{ps}}^{2}(x) \approx\langle H(x ; 0)\rangle_{\mathrm{ps}}+\langle H(x ; 0)\rangle_{\mathrm{ps}}^{2} \frac{\langle 1-n\rangle}{\langle n\rangle},
$$

where the second term is due to promoter switching with variance $\langle 1-n\rangle\langle n\rangle$, and the first term describes the Poisson noise coming from the production, diffusion, and decay of $\mathrm{Hb}$ when the promoter is active.

Figure 4(a) shows $\sigma_{\mathrm{ps}}^{2}(x)$ for different values of $\lambda$. For small $\lambda, \sigma_{\mathrm{ps}}^{2}(x)$ is large for small $x$, but reduces quickly with increasing $x$. Hence, the variance at a given nucleus is determined by few nuclei in the neighborhood but each contribution is large. For increasing $\lambda$ the number of nuclei contributing to the variance increases, but the contributions of nearby nuclei are smaller; this is because diffusion washes out the bursts of $\mathrm{Hb}$ production at the source. To see which of the two opposing effects dominates, we integrate Eq. (6) to obtain the total variance:

$$
\sigma^{2}=\langle H\rangle+\frac{\langle H\rangle^{2}}{4(\lambda / \ell)} \frac{\langle 1-n\rangle}{\langle n\rangle} .
$$

Compared to the variance in an isolated nucleus, the overall effect of diffusion in a one-dimensional, uniform space is a reduction of the non-Poissonian noise by $(4 \lambda / \ell)^{-1}$. The Poissonian part cannot be reduced because diffusion itself is Poissonian. Indeed, spatial averaging can reduce the effect of noise in gene expression, but only if this is super-Poissonian.

The finite range of non-Poissonian fluctuations implies the following model. $N$ nuclei contribute equally to a well-
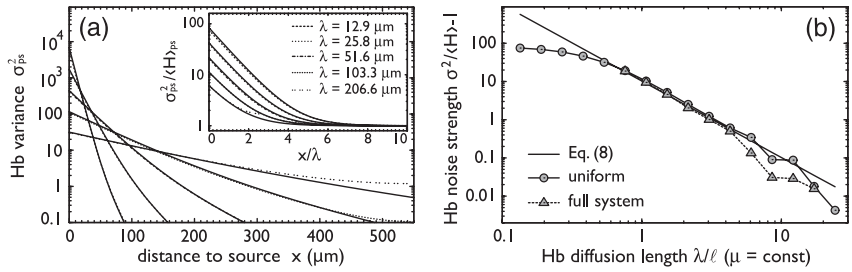

FIG. 4. (a) Variance $\sigma_{\mathrm{ps}}^{2}(x)$ of $\mathrm{Hb}$ as function of the distance $x$ from a point source at $x=0$ in one-dimensional space for increasing $\mathrm{Hb}$ diffusion length $\lambda$. Equation (6) is compared to simulation results (dashed lines) for $\mu=1.2 \times 10^{-2} \mathrm{~s}^{-1}$ and $\beta=500 \mu=6 \mathrm{~s}^{-1}$, i.e., for fast $\mathrm{Hb}$ dynamics; $\lambda$ is varied through the diffusion coefficient $D$. The inset shows the $\mathrm{Hb}$ noise strength $\sigma_{\mathrm{ps}}^{2}(x / \lambda) /\langle H(x / \lambda ; 0)\rangle_{\mathrm{ps}}$ as a function of $x / \lambda$. (b) Non-Poissonian part of the Hb noise strength, $\sigma^{2} /\langle H\rangle-1$, for a uniform system with $N=32 \times 32$ nuclei on a square lattice as function of $\lambda / \ell ; D$ is varied, $\langle n\rangle=0.5, \beta$ and $\mu$ are constant as in Fig. 1 [Hb dynamics is thus not fast, as in panel (a)]. Simulation results for the uniform system (circles) are compared to Eq. (8) with $N(\lambda)=8(\lambda / \ell)^{2}$ (solid line). The value $\sigma_{0}^{2} /\langle H\rangle-1=82.4$ [see Eq. (8)] in an isolated volume has been calculated numerically. These results are compared to the $\mathrm{Hb}$ noise strength for the full, two-dimensional system (see Fig. 1) in a nucleus at the boundary with $\langle n\rangle=0.5$ (triangles). 
stirred reservoir containing $M \mathrm{Hb}$ molecules, with $\langle M\rangle=$ $N\langle H\rangle$ and variance $\sigma_{M}^{2}=N \sigma_{0}^{2}$, where $\sigma_{0}^{2}$ is the noise of $H$ in a nucleus if there were no $\mathrm{Hb}$ diffusion. Each nucleus then samples $\mathrm{Hb}$ molecules in a binomial fashion from this reservoir. The noise in each nucleus is given by $\sigma^{2}=$ $E[V[H \mid M]]+V[E[H \mid M]]$, where $E[X]$ denotes the expectation and $V[X]$ the variance of $X$. The first term describes the noise in the sampling process, and is given by $E[V[H \mid M]]=\langle H\rangle\left(1-N^{-1}\right)$. The second term describes the variance in $H$ due to fluctuations in the reservoir, and is given by $V[E[H \mid M]]=\sigma_{0}^{2} / N$. Hence,

$$
\sigma^{2}=\langle H\rangle+\frac{\sigma_{0}^{2}-\langle H\rangle}{N} .
$$

This shows that it is the super-Poissonian part of the noise, $\sigma_{0}^{2}-\langle H\rangle$, which is reduced by spatial averaging. Since $N$ scales with the dimensionality $d$ as $N \sim \lambda^{d}$, this reduction becomes more efficient in higher dimensions. Interestingly, Eq. (8) also reveals that if $h b$ expression would be sub-Poissonian [8], e.g., due to active mRNA or protein degradation, diffusion would increase the noise.

Figure 4(b) shows the non-Poissonian part of the variance in a uniform, two-dimensional system as function of $\lambda$; the exact, numerical result is compared to Eq. (8) with $N$ chosen to be the number of nuclei within a distance $2 \lambda$ of a given nucleus along the edges of a square lattice, $N(\lambda)=$ $8(\lambda / \ell)^{2}$. For sufficiently large $\lambda$ for which $N(\lambda)>1$, the approximation is excellent. Figure 4(b) also compares the results for the uniform system to those of the full, nonuniform system in which a Bcd gradient activates Hb. Clearly, the well-stirred approximation describes $\sigma^{2}$ at the $\mathrm{Hb}$ boundary very well; only for $\lambda>5 \ell$ is the variance at the boundary significantly influenced by nuclei far from the boundary with smaller variance.

Finally, we can combine Eqs. (5) and (8) with Eq. (3) to predict the boundary width $\Delta x$. Figure 2(b) shows that the prediction agrees very well with the simulations. This figure also shows that the two antagonistic effects of $\mathrm{Hb}$ diffusion-reducing the slope but also the variance-lead to a diffusion constant that optimizes the boundary width for a fixed $\mathrm{Hb}$ lifetime. Interestingly, this minimal width is less than one nuclear spacing, as found experimentally [2]. This suggests that for achieving the necessary precision, mechanisms based on multiple gradients or interactions between multiple Bcd targets are not required, although these may provide robustness against embryo-to-embryo variations [9-11]. Since the exact mechanism for $\mathrm{Hb}$ transport is unknown, it is tempting to speculate that the precision achieved via spatial averaging could be increased further by separating the two effects of $\mathrm{Hb}$ diffusion using an anisotropic transport of $\mathrm{Hb}$ : slow transport along the anterior-posterior axis would allow for steep spatial profiles, while fast diffusion along the perpendicular direction would allow for effective spatial averaging. The benefit of anisotropic transport could imply the existence of active mechanisms for $\mathrm{Hb}$ transport.

Spatial averaging can only be beneficial when the noise in $\mathrm{Hb}$ production has a super-Poissonian component. The observation of spatial correlations in Hb indicates that this is the case [2,12]. The question arises whether bursts in gene expression are inevitable and spatial averaging a prerequisite for achieving a precise $h b$ expression domain. $\mathrm{The} \mathrm{Hb}$ lifetime is limited by the nuclear division time, while the time scale for promoter state fluctuations is limited by the diffusion of Bcd. The magnitude of the bursts could be reduced by reducing the promoter strength, but this would lower the $\mathrm{Hb}$ copy number and hence the steepness of the Hb boundary; the net result would, in fact, be a decrease of its precision. In a model with explicit $h b$ mRNA, the effect of promoter state fluctuations could be alleviated by reducing the transcription rate. However, to achieve a sufficiently steep and precise $\mathrm{Hb}$ boundary with a low copy number of $h b$ mRNA, the translation rate had to be increased such that $\mathrm{Hb}$ production becomes superPoissonian again. Moreover, $\mathrm{Hb}$ autoactivates its own expression [13], which also tends to increase the superPoissonian part of the noise. It thus appears that spatial averaging is a fundamental mechanism for generating precise patterns of gene expression. Clearly, it will be of interest to study how other modes of transport, e.g., subdiffusion or active transport, affect the mechanism of spatial averaging.

We thank Filipe Tostevin for a critical reading of the manuscript. This work is supported by FOM/NWO (T. E., P. R. t. W.) and The Royal Society (M.H.).

[1] B. Houchmandzadeh, E. Wieschaus, and S. Leibler, Nature (London) 415, 798 (2002).

[2] T. Gregor, D. W. Tank, E. F. Wieschaus, and W. Bialek, Cell 130, 153 (2007).

[3] F. He et al., Dev. Cell 15, 558 (2008).

[4] Y. Okabe-Oho, H. Murakami, S. Oho, and M. Sasai, PLoS Comput. Biol. 5, e1000486 (2009).

[5] T. Gregor et al., Cell 130, 141 (2007).

[6] J. Elf and M. Ehrenberg, Syst. Biol. 1, 230 (2004).

[7] F. Tostevin, P. R. ten Wolde, and M. Howard, PLoS Comput. Biol. 3, e78 (2007).

[8] J. M. Pedraza and J. Paulsson, Science 319, 339 (2008).

[9] M. Howard and P. R. ten Wolde, Phys. Rev. Lett. 95, 208103 (2005).

[10] Manu et al., PLoS Biol. 7, e1000049 (2009).

[11] Manu et al., PLoS Comput. Biol. 5, e1000303 (2009).

[12] G. Tkačik, T. Gregor, and W. Bialek, PLoS ONE 3, e2774 (2008).

[13] J. Treisman and C. Desplan, Nature (London) 341, 335 (1989). 